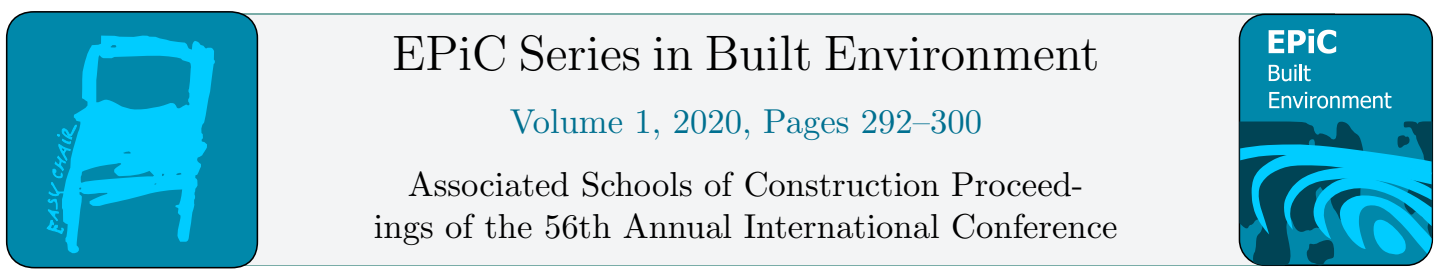

\title{
Point-Cloud Models of Historical Barns - Spatial Discrepancies of Laser Scanning versus Robotic Total Station
}

\author{
Gustavo Maldonado ${ }^{1}$, Marcel Maghiar ${ }^{1}$, Brent Tharp ${ }^{1}$ and Dhruv Patel ${ }^{2}$ \\ ${ }^{1}$ Georgia Southern University, Statesboro, GA, USA \\ ${ }^{2}$ Georgia Department of Transportation, Savannah, GA, USA \\ gmaldonado@georgiasouthern.edu, mmaghiarlgeorgiasouthern.edu \\ btharpegeorgiasouthern.edu, dpateledot.ga.gov
}

\begin{abstract}
This study considers the generation of virtual, 3D point-cloud models of seven deteriorating historical, agricultural barns in Bulloch County, Georgia, USA, for preservation purposes. The work was completed as a service-learning project in a course on Terrestrial Light Detection and Ranging (T-LiDAR), offered at Georgia Southern University. The resulting models and fly-through videos were donated to Bulloch County Historical Society and to the Georgia Southern Museum, to make them available to the general public and future generations. Additionally, one of the seven barns was selected to be extensively measured to estimate the relative spatial accuracy of all seven resulting 3D point-cloud models, with respect to measurements completed with a highly accurate instrument. Three accurate benchmarks were established around it for georeferencing purposes. The positions of 44 points were measured in the field via an accurate, onesecond, robotic total-station (RTS) instrument. Also, the coordinates of the same points were acquired from within georeferenced and non-georeferenced point-cloud models. These points defined 259 distances. They were compared to determine their discrepancy statistics. It was observed that this process produced virtual models with an approximate maximum spatial discrepancy of one-half inch $(0.5 \mathrm{in})$ with respect to measurements performed by a highly accurate RTS device. There were no substantial differences in the relative accuracies of the georeferenced and non-georeferenced models.
\end{abstract}

\section{Introduction, Objective and Literature Review}

The main objective of this project is two-fold: (i) Generation of three-dimensional (3D) point-cloud, virtual models of several deteriorating, agricultural barns in Bulloch County, GA, USA, via T-LiDAR techniques, for historical preservation purposes. (ii) Analysis of spatial discrepancies, attained within 
the resulting model of one barn, versus measurements completed in the field by a highly accurate, onesecond, RTS device.

Even though instrument manufacturers publicize the accuracy of their instruments, it is known that the actual accuracies attained under diverse field conditions, not necessarily coincide with the indicated ones. These differences motivated the completion of this discrepancy analysis. The obtained statistical result assists history and preservation professionals in estimating dimensional spatial errors in the resulting 3D point-cloud models of similar structures. Since all involved barns are structures with relatively similar size, age, materials, and the same modeling technique was employed in all of them, it is expected the results of the present discrepancy analysis, on one of the barns, could be extended to the models of all seven of them to approximately estimate their relative spatial accuracies, with respect to measurements taken, in the field, by an accurate RTS instrument.

T-LiDAR, or laser scanning, is a remote sensing technique that digitally captures the shape of spatial objects using beams of laser light. These scanners are modern and powerful surveying instruments able to determine the spatial location of millions of points hit by rapidly produced laser beams. 3D Laser Scanners create dense point clouds of data from the surface of objects. In particular, the laser-based scanner employed in this project can capture the location of up to 50,000 points per second. Each captured point contains seven numbers: three spatial coordinates (x, y, z); three color coordinates (red, green, blue); and the intensity of the light reflected back to the instrument from the hit point. Once all the data is collected, it is then processed by the Cyclone software package from Leica Geosystems. This software is employed to convert the raw data, gathered in the field, into final 3D models.

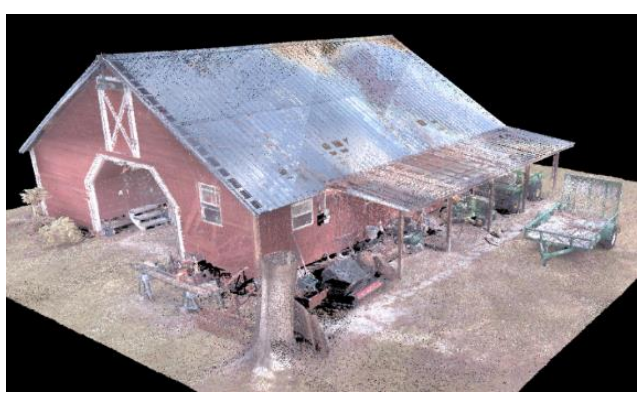

(a) Front and side view

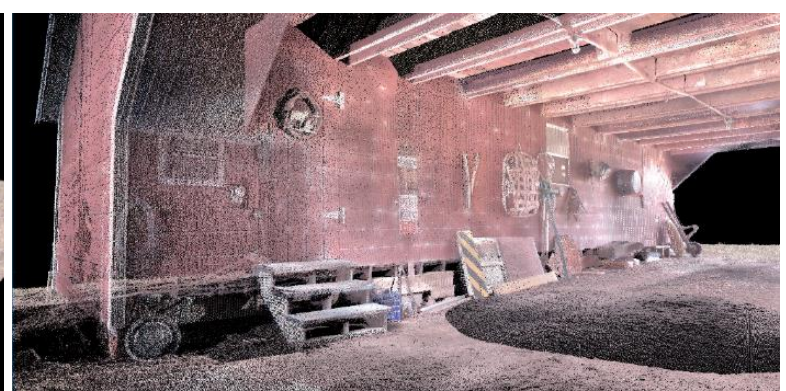

(b) Internal view

Figure 1: Resulting point-cloud model of pilot barn

The work was completed as a project assignment in an Introduction to T-LiDAR course, offered by the Department of Civil Engineering and Construction of Georgia Southern University. This is a 5000level elective course for undergraduate and graduate students in three different programs, Construction Management, Civil Engineering, and Construction Engineering. The project involved seven barns. The first one was initially completed by the first author and a graduate assistant, Mariah Peart, as a pilot activity, to confirm that this was a potentially attainable project by three groups (of three students each), during the 15 weeks of a regular academic semester. Figures 1(a) and 1(b) show screen-captured images of the resulting pilot barn completed in April 2018. The property belongs to the Akins family and is located near Stilson, in Bulloch County, Georgia, USA. All six remaining modeled barns are also located in the same County.

In 2008, an article on the evaluation of 3D laser scanning for highway construction applications was published (Slattery et al., 2008). It concluded that "Preliminary results indicate it is feasible to use laser scanning technology to accurately map terrain prior to road construction." A 2011 study (Holley et al., 2011) explored the accuracy of reflectorless electronic distance measuring devices and showed that the proper use of these devices did not affect accuracy considerably for relatively short distances when compared against the use of reflecting prisms. Previous studies, similar to the current one, (Maldonado 
et al., 2015; Newsome, 2016) compared discrepancies between measurements completed on and nearby buildings. They used the same scanner employed in this work, but a less accurate (7-second) totalstation instrument. Also, in other study completed by Maldonado et al. (2018), discrepancies were determined between the same scanner and the same RTS device used in this project while measuring a complex street intersection. The work from 2015 used an 11-sided traverse for georeferencing purposes and involved a building with plant size of $155 \mathrm{ft} \times 290 \mathrm{ft}$. On the other hand, the work from 2018 employed a Global Navigation Satellite System (GNSS) device for ground control and covered an area of about $300 \mathrm{ft} \times 750 \mathrm{ft}$. The area covered in the current project is smaller than in those previous cases and the current georeferencing approach is different, but still close to the one employed in the 2015 project. In none of those cases, comparisons were made between distances extracted from georeferenced and non-georeferenced models. However, a recently publicized Master Thesis by Mariah Peart (2019) presents comparisons between those two types of models. Recently, a study on the accuracy assessment of mine walls was published (Long et al., 2018). It used LiDAR points to generate surfaces using nonuniform rational basis splines. The accuracy of the resulting surfaces was assessed. However, the current study uses only points (not fitted surfaces) to obtain position and distance measurements form within the final virtual point-cloud model. That is, the current discrepancy study is based on minimum postprocessing.

\section{Instruments and Methodology}

The laser-based scanner, employed in this project, is the Leica Geosystem ScanStation C10. A second instrument, also from Leica Geosystems, the robotic station TCRP 1201+, served as the benchmarking device, due to its high accuracy. A previous article, by two of the current authors, includes a table comparing the main characteristics and capabilities of both instruments (Maldonado et al., 2018).

The methodology consisted in assigning two barns to each group of three students and swiftly scan their exteriors and interiors, minimizing the time in the field by not capturing registration (stitching) targets. That is, different scans of the same barn were stitched together (registered in the same system of reference) via Leica's Visual Alignment technique. This approach is not automatic and requires human intervention to successively stitch two scans at a time, in a computer laboratory. A total of seven barns were fully scanned and one of them was selected, as a representative one, to estimate the relative accuracy of its resulting point-cloud model. The selected barn was assigned to be processed by a group of three graduate students. This barn is the closest one to the Statesboro Campus of Georgia Southern University and is located on the grounds of the Georgia Southern Botanic Garden. Three ground control points were accurately established around the selected building, via a combined trilateration and closedtraverse procedure. This resulted in a total angular error of closure of 22.4 seconds and, after balancing errors, the attained longitudinal precision was 1 in 21,370 which is equivalent to $0.0047 \mathrm{ft}$ (or $0.056 \mathrm{in}$, or $1.4 \mathrm{~mm}$ ) in $100 \mathrm{ft}$. For georeferencing purposes, the scans captured one target on of each of these three ground points (benchmarks). After the final point-cloud model was generated, it was georeferenced using the accurately determined coordinates of those three benchmarks.

The coordinates of 44 points were obtained in the field using the accurate, one-second, RTS instrument. The coordinates of the same points were also extracted from within the point-cloud model. They were compared to calculate position discrepancies. Also, five of those points were designated as center points (CPs) and distances were measured from those CPs to all remaining points. This resulted in 259 calculated distances for comparison purposes. Additionally, the same distances were also measured within a non-georeferenced model, of the same barn, to study the effect of georeferencing in the attained discrepancies. 


\section{Results}

This work generated virtual, 3D, point-cloud models of seven (7) old and deteriorating barns in Bulloch County, Georgia, for historical preservation purposes. All seven structures were similar in size and the same modeling technique, terrestrial LiDAR (or laser scanning), was employed in all cases. Additionally, one of the modeled barns $(\approx 42 \mathrm{ft} \times 59 \mathrm{ft})$, located at the Georgia Southern University Botanic Garden, was selected to perform a discrepancy analysis to estimate the accuracy of the resulting 3D models of all seven barns. This analysis consisted in comparing point positions and distances, between virtual cloud points, versus positions and distances of the same points, but measured in the field (real points). These field positions and distances were measured with a highly accurate RTS instrument, considered as the benchmarking device in this study. The virtual distances were extracted from two different point-cloud models. One was non-georeferenced and other was georeferenced via three accurately established ground points (or benchmarks). This allowed to observe the effect of georeferencing in those discrepancies. Positions and distances extracted from the non-georeferenced point-cloud model are referred herein as non-georef values, whereas those extracted from the georeferenced point-cloud model are referred to as georef values. Positions and distances measured in the field, with the accurate RTS device, are indicated as field values.

Forty-four (44) point positions were initially measured. Several of them were marked on the walls of the barn and a few in the surrounding areas. Six points were selected as center points and distances were measured from these CPs to all remaining points. This generated a total of 259 distances, ranging in length from 3 to 132 feet. They were measured and compared as indicated in the previous paragraph. A couple of points presented large positional discrepancies and they were identified as outliers in this study. They could have been wrongly acquired in the field, wrongly annotated in the field book or wrongly identified in the point cloud. Once the two outliers were removed, this work considered 42 points and 247 distances. The discrepancy statistics for the position of those points are presented in Table 1. It is observed that the Root Mean Square (RMS) value of the Northing coordinates was reduced from $0.017 \mathrm{ft}$ to $0.014 \mathrm{ft}$, after the removal of the two outliers. Nevertheless, all calculations and graphs were performed with and without outliers to notice their effect.

Table 1: Statistics for discrepancies in point positions - Georef model vs field values.

\begin{tabular}{|c|c|c|c|c|c|c|}
\hline & \multicolumn{3}{|c|}{44 Points (with 2 outliers) } & \multicolumn{3}{|c|}{42 Points (without outliers) } \\
\hline & $\begin{array}{l}\text { Northing } \\
\text { (ft) }\end{array}$ & $\begin{array}{l}\text { Easting } \\
\text { (ft) }\end{array}$ & $\begin{array}{l}\text { Elevation } \\
\text { (ft) }\end{array}$ & $\begin{array}{l}\text { Northing } \\
\text { (ft) }\end{array}$ & $\begin{array}{c}\text { Easting } \\
\text { (ft) }\end{array}$ & $\begin{array}{c}\text { Elevation } \\
\text { (ft) }\end{array}$ \\
\hline Mean Values & 0.001 & 0.000 & -0.004 & -0.000 & -0.000 & -0.004 \\
\hline Max Values & 0.070 & 0.024 & 0.050 & 0.029 & 0.024 & 0.050 \\
\hline Min Values & -0.021 & -0.039 & -0.042 & -0.021 & -0.039 & -0.042 \\
\hline STD.P & 0.017 & 0.012 & 0.017 & 0.014 & 0.012 & 0.017 \\
\hline RMS Values & 0.017 & 0.012 & 0.017 & 0.014 & 0.012 & 0.017 \\
\hline
\end{tabular}

Similarly, the discrepancies in distances were determined with and without outliers. Virtual distances were extracted from both 3D point-cloud models, georef and non-georef ones. The calculated statistics of the discrepancies included minimum, maximum, mean, standard deviations and RMS values. STD.P indicates the standard deviation of the population. It was calculated using the corresponding function in Microsoft Excel. In all results, it is observed that the STD.P and RMS values are close in magnitude. This is because the corresponding mean discrepancy values are close to zero. Additionally, the cumulative distribution functions of the discrepancies, in distances, were obtained. Their graphs assisted in visualizing the performance of the georef and non-georef models. 
Comparison distances were calculated from each of five selected CPs to the remaining points. Each $\mathrm{CP}$ was chosen so their location discrepancies, between georef and field values, were different. Those differences ranged from $0.008 \mathrm{ft}$ to $0.072 \mathrm{ft}$. The selected CPs are those identified as: 2-01, 2-09, 2-18, 3-05, 3-15 and benchmark TG 1 . These CPs remained the same during all comparisons, involving georef, non-georef data, with and without outlying points. The statistics (Mean, Max, Min, STD P, and RMS values) corresponding to discrepancies in distances, between georef and field values, are presented in Table 2. On the other hand, Table 3 shows the same statistics after removing the two outlying points.

Table 2: Analysis of discrepancies in distances - Georef model vs field values (with outliers)

\begin{tabular}{|c|c|c|c|c|c|c|c|c|}
\hline $\begin{array}{c}\text { Center } \\
\text { Point }\end{array}$ & $\begin{array}{l}\text { \# of } \\
\text { Dist. }\end{array}$ & $\begin{array}{c}\text { Mean } \\
\text { Discr }(f t)\end{array}$ & $\begin{array}{c}\text { STD P } \\
\text { Discr }(f t)\end{array}$ & $\begin{array}{c}\text { RMS Value } \\
\text { Discr (ft) }\end{array}$ & $\begin{array}{c}\text { Min } \\
\mid \text { Discr } \mid(f t)\end{array}$ & $\begin{array}{c}\text { Max } \\
\mid \text { Discr } \mid(f t)\end{array}$ & $\begin{array}{c}\text { Mean } \\
\mid \text { Discr } \mid(f t)\end{array}$ & $\begin{array}{c}\text { STD P } \\
\mid \text { Discr } \mid(f t)\end{array}$ \\
\hline TG1 & 44 & -0.0026 & 0.0167 & 0.0169 & 0.0007 & 0.0509 & 0.0135 & 0.0102 \\
\hline 2-09 & 43 & 0.0006 & 0.0142 & 0.0142 & 0.0006 & 0.0402 & 0.0107 & 0.0093 \\
\hline $2-18$ & 43 & 0.0020 & 0.0176 & 0.0178 & 0.0011 & 0.0106 & 0.0143 & 0.0106 \\
\hline $2-01$ & 43 & 0.0036 & 0.0158 & 0.0162 & 0.0014 & 0.0094 & 0.0132 & 0.0094 \\
\hline $3-15$ & 43 & -0.0028 & 0.0128 & 0.0131 & 0.0001 & 0.0091 & 0.0094 & 0.0091 \\
\hline 3-05 & 43 & -0.0112 & 0.0153 & 0.0189 & 0.0001 & 0.0136 & 0.0131 & 0.0136 \\
\hline
\end{tabular}

Table 3: Analysis of discrepancies in distances - Georef model vs field values (without outliers)

\begin{tabular}{ccccccccc}
\hline $\begin{array}{c}\text { Center } \\
\text { Point }\end{array}$ & \# of & $\begin{array}{c}\text { Mean } \\
\text { Dist. }\end{array}$ & $\begin{array}{c}\text { Discr }(f t) \\
\text { STD P }\end{array}$ & $\begin{array}{c}\text { RMS Value } \\
(f t)\end{array}$ & $\begin{array}{c}\text { Miscr }(f t) \\
\mid \text { Discr } \mid(f t)\end{array}$ & $\begin{array}{c}\text { Max } \\
\mid \text { Discr } \mid(f t)\end{array}$ & $\begin{array}{c}\text { Mean } \\
\mid \text { Discr } \mid(f t)\end{array}$ & $\begin{array}{c}\text { STD P } \\
\mid \text { Discr } \mid(f t)\end{array}$ \\
\hline TG1 & 42 & -0.0039 & 0.0149 & 0.0154 & 0.0007 & 0.0297 & 0.0129 & 0.0085 \\
$2-09$ & 41 & 0.0009 & 0.0144 & 0.0145 & 0.0006 & 0.0402 & 0.0109 & 0.0095 \\
$2-18$ & 41 & 0.0033 & 0.0156 & 0.0160 & 0.0011 & 0.0085 & 0.0135 & 0.0085 \\
$2-01$ & 41 & 0.0036 & 0.0149 & 0.0155 & 0.0014 & 0.0088 & 0.0128 & 0.0088 \\
$3-15$ & 41 & -0.0018 & 0.0115 & 0.0116 & 0.0001 & 0.0076 & 0.0088 & 0.0076 \\
$3-05$ & 41 & -0.0093 & 0.0108 & 0.0143 & 0.0001 & 0.0087 & 0.0113 & 0.0087 \\
\hline
\end{tabular}

Table 4: Analysis of discrepancies in distances - Non-georef model vs field values (with outliers)

\begin{tabular}{|c|c|c|c|c|c|c|c|c|}
\hline $\begin{array}{c}\text { Center } \\
\text { Point }\end{array}$ & $\begin{array}{l}\# \text { of } \\
\text { Dist. }\end{array}$ & $\begin{array}{c}\text { Mean } \\
\text { Discr }(f t)\end{array}$ & $\begin{array}{c}\text { STD P } \\
\operatorname{Discr}(f t)\end{array}$ & $\begin{array}{c}\text { RMS Value } \\
\text { Discr }(f t)\end{array}$ & $\begin{array}{c}\text { Min } \\
\mid \text { Discr } \mid(f t)\end{array}$ & $\begin{array}{c}\text { Max } \\
\mid \text { Discr } \mid(f t)\end{array}$ & $\begin{array}{c}\text { Mean } \\
\mid \text { Discr } \mid(f t)\end{array}$ & $\begin{array}{c}S T D P \\
\mid \text { Discr } \mid(f t)\end{array}$ \\
\hline TG1 & 44 & -0.0105 & 0.0264 & 0.0284 & 0.0010 & 0.1097 & 0.0194 & 0.0208 \\
\hline $2-09$ & 43 & -0.0238 & 0.0377 & 0.0446 & 0.0000 & 0.1774 & 0.0301 & 0.0329 \\
\hline $2-18$ & 43 & 0.0077 & 0.0335 & 0.0344 & 0.0011 & 0.0280 & 0.0200 & 0.0280 \\
\hline $2-01$ & 43 & 0.0022 & 0.0234 & 0.0235 & 0.0022 & 0.0162 & 0.0170 & 0.0162 \\
\hline $3-15$ & 43 & 0.0038 & 0.0369 & 0.0371 & 0.0001 & 0.0323 & 0.0182 & 0.0323 \\
\hline $3-05$ & 43 & -0.0090 & 0.0413 & 0.0423 & 0.0033 & 0.0326 & 0.0270 & 0.0326 \\
\hline
\end{tabular}


Tables 4 and 5 present the statistics of distance discrepancies between the non-georef model and field measurements, with and without outliers, respectively. As expected, the standard deviations of the set without outliers indicate less dispersion.

Table 5: Analysis of discrepancies in distances - Non-georef model vs field values (without outliers)

\begin{tabular}{|c|c|c|c|c|c|c|c|c|}
\hline $\begin{array}{l}\text { Center } \\
\text { Point }\end{array}$ & $\begin{array}{l}\text { \# of } \\
\text { Dist. }\end{array}$ & $\begin{array}{c}\text { Mean } \\
\operatorname{Discr}(f t)\end{array}$ & $\begin{array}{c}\text { STD P } \\
\operatorname{Discr}(f t)\end{array}$ & $\begin{array}{c}\text { RMS Value } \\
\text { Discr }(f t)\end{array}$ & $\begin{array}{c}\text { Min } \\
\mid \text { Discr } \mid(f t)\end{array}$ & $\begin{array}{c}\text { Max } \\
\mid \text { Discr } \mid(f t)\end{array}$ & $\begin{array}{c}\text { Mean } \\
\mid \text { Discr } \mid(f t)\end{array}$ & $\begin{array}{c}\text { STD P } \\
\mid \text { Discr } \mid(f t)\end{array}$ \\
\hline TG1 & 42 & -0.0113 & 0.0146 & 0.0185 & 0.0010 & 0.0497 & 0.0154 & 0.0102 \\
\hline $2-09$ & 41 & -0.0214 & 0.0289 & 0.0360 & 0.0000 & 0.1290 & 0.0265 & 0.0244 \\
\hline $2-18$ & 41 & 0.0087 & 0.0323 & 0.0335 & 0.0011 & 0.0278 & 0.0186 & 0.0278 \\
\hline $2-01$ & 41 & 0.0050 & 0.0174 & 0.0181 & 0.0022 & 0.0099 & 0.0151 & 0.0099 \\
\hline $3-15$ & 41 & 0.0037 & 0.0284 & 0.0286 & 0.0001 & 0.0252 & 0.0136 & 0.0252 \\
\hline $3-05$ & 41 & -0.0090 & 0.0340 & 0.0352 & 0.0033 & 0.0269 & 0.0227 & 0.0269 \\
\hline
\end{tabular}

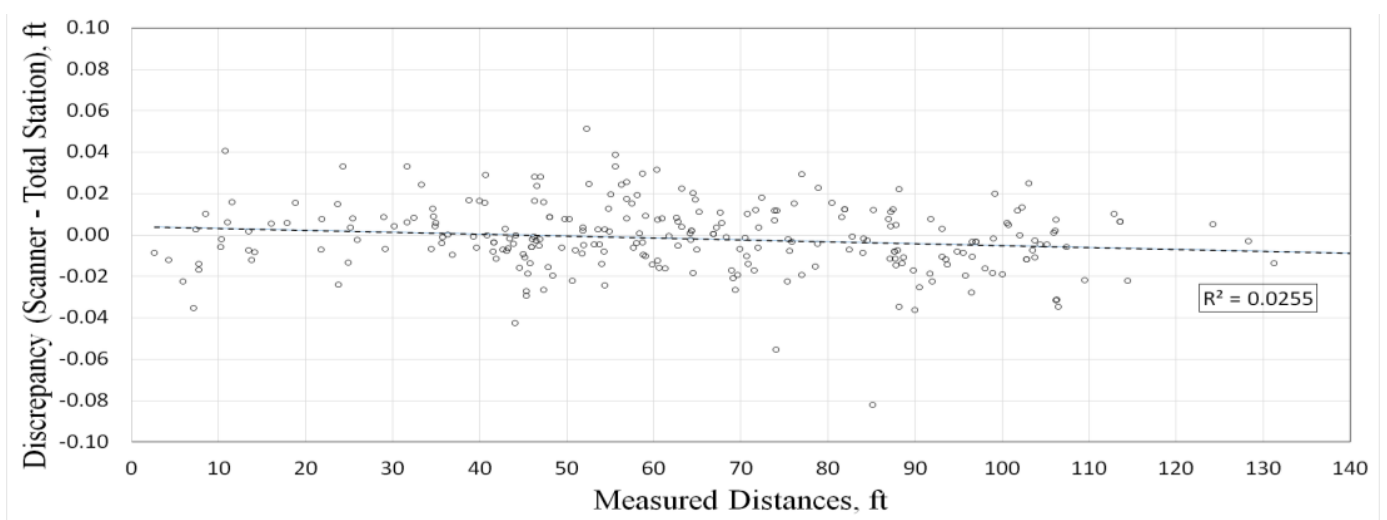

Figure 2: Discrepancies in 259 calculated distances - Georef vs field values (with outliers)

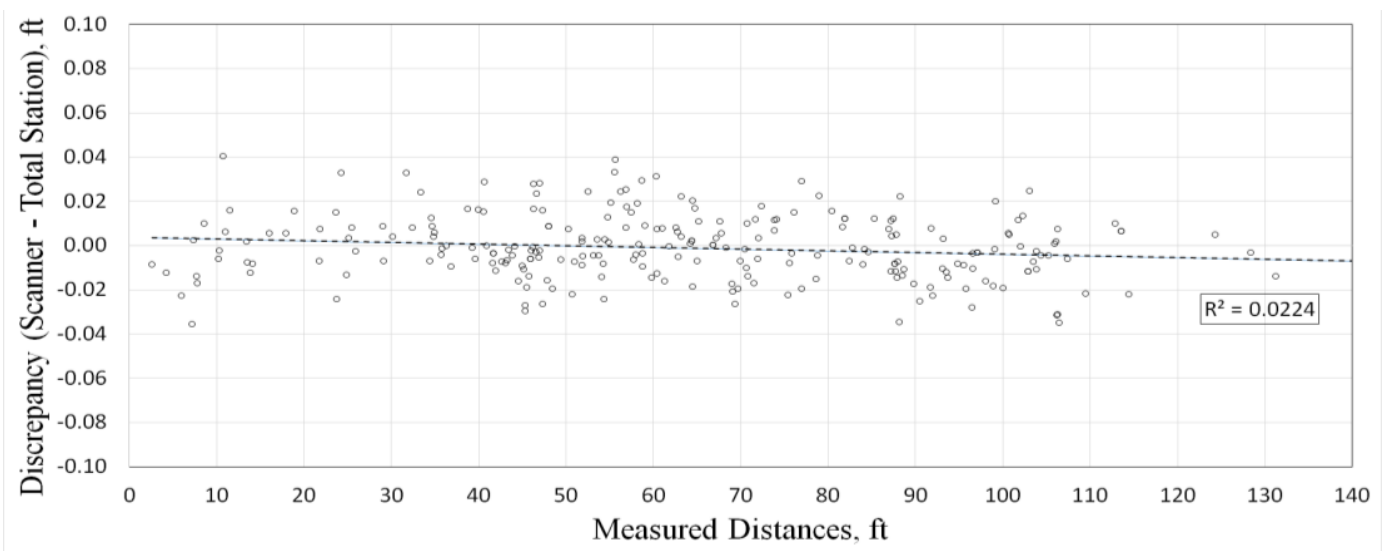

Figure 3: Discrepancies in 247 calculated distances - Georef vs field values (without outliers) 
Figures 2 and 3 depict distance discrepancies vs measured lengths. Respectively, they compare distances in the georef model vs field measurements, with and without outliers.

Similarly, Figures 4 and 5 show discrepancy statistics for distances measured in the non-georef model vs those measured in the field, with and without outliers, respectively. In these figures, it is observed that discrepancies in distances are not related to the actual length of the measured distance. They are almost uniformly distributed along the analyzed distance range, 3 to 132 feet. This is corroborated by the low $\mathrm{R}^{2}$ value (square of the correlation coefficient, $\mathrm{r}$ ) shown in the graphs.

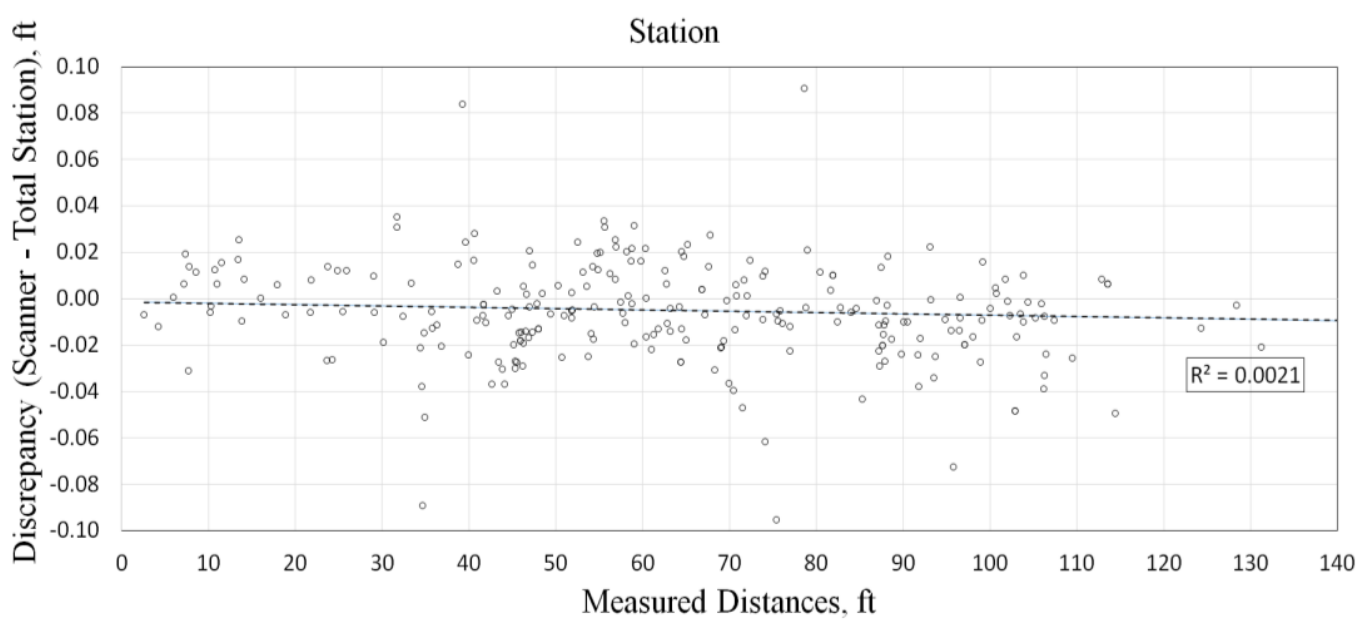

Figure 4: Discrepancies in 259 calculated distances - Non-georef vs field values (with outliers)

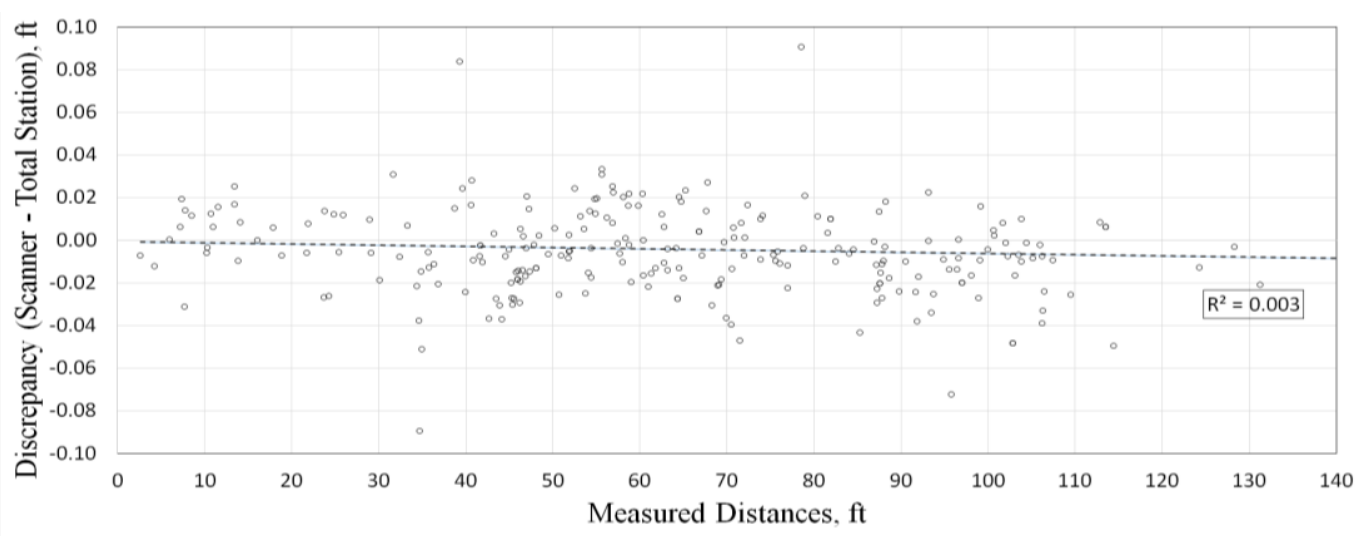

Figure 5: Discrepancies in 247 calculated distances - Non-georef vs field values (without outliers) 


\section{Conclusions, Closing Remarks and Acknowledgments}

The point-cloud models of six deteriorating barns were successfully generated during a semesterlong course, attended by nine students, six undergraduate and three graduate ones. A pilot point-cloud model, for a seventh barn, had previously been generated by the instructor and a graduate assistant. This project was completed as a service-learning activity and the resulting models were donated to the Bulloch County Historical Society and to the Georgia Southern Museum.

One barn, located near the Georgia Southern University Campus, was selected to study the relative spatial accuracy attained within its virtual, 3D, point-cloud model, with respect to measurements completed via an accurate (one second) robotic total-station instrument. All position and distance measurement comparisons were performed via Microsoft Excel. They included the following six cases:

(1) Comparison of georef positions (with outliers): The position discrepancy of 44 points (including 2 outliers) were considered by using the 68-95-99.7 rule of a normal distribution. In the $\pm \sigma$ band, $68 \%$ of the positions have a discrepancy within $\pm 0.017 \mathrm{ft}( \pm 0.204 \mathrm{in}$.), in the $\pm 2 \sigma$ band, $95 \%$ of the discrepancies are within $\pm 0.034 \mathrm{ft}( \pm 0.408 \mathrm{in}$.), and in the $\pm 3 \sigma$ band, $99.7 \%$ are within $\pm 0.051 \mathrm{ft}$ (0.612 in.). That is, almost all point positions extracted from the georeferenced point cloud show discrepancies equal or less than 0.612 in $[15.5 \mathrm{~mm}]$ with respect to the same positions, measured in the field, with the accurate, one-second, RTS instrument.

(2) Comparison of distances - Georef vs field values (with outliers): Data from the georef point cloud, with outliers, present discrepancy RMSV $= \pm 0.018 \mathrm{ft}( \pm 0.21 \mathrm{in})$, with most distances $(98.1 \%)$ having a discrepancy in the band $\pm 0.040 \mathrm{ft}( \pm 0.48 \mathrm{in})$.

(3) Comparison of distances - Georef vs field values (without outliers): Similarly, without outliers, the discrepancies between the georef model and field measurements show RMSV $= \pm 0.016 \mathrm{ft}( \pm 0.20$ in), with most distances $(99.6 \%)$ presenting discrepancies within the $\pm 0.04 \mathrm{ft}( \pm 0.48$ in) band.

(4) Comparison of distances - Non-georef vs field values (with outliers): Data from the non-georef point cloud, with outliers, present discrepancy RMSV $= \pm 0.036 \mathrm{ft}( \pm 0.43 \mathrm{in})$, with most distances $(90.7 \%)$ having discrepancies in the $\pm 0.04 \mathrm{ft}$ ( $\pm 0.48 \mathrm{in})$ band.

(5) Comparison of distances - Non-georef vs field values (without outliers): Similarly, without outliers, the discrepancies between the non-georef model and field measurements show RMSV $= \pm 0.016 \mathrm{ft}( \pm 0.20 \mathrm{in})$, with most distances $(93.9 \%)$ having discrepancies in the $\pm 0.04 \mathrm{ft}$ $( \pm 0.48$ in) band.

(6) Comparison of distances - Non-georef and georef vs field values (without outliers): It is graphically observed in Figure 6, that the accurately georeferenced model did not add errors to those presented by the non-georef data, both with respect to field measurements. On the contrary, in this case the georef model presents less discrepancy than the non-georef model.

Therefore, for the range of distances considered in this study (3-132 feet), it is observed that the 3D point-cloud model of the analyzed barn presents spatial discrepancies with a maximum magnitude of approximately half inch $(\sim 13 \mathrm{~mm})$, with respect to distance measurements completed with a highly accurate robotic total-station device. Since the other six barns were relatively similar in size, and were 
modeled using the same T-LiDAR instrument and procedure, it is expected that their spatial discrepancies are also similar to the above mentioned one, with a maximum value near half inch.

The authors express their sincere gratitude to former graduate research assistant, Mariah D. Peart, to former graduate students, Keertan Akshar and Ananya Augustine, and to former undergraduate students, Thomas L. Graham, Benjamin A. Moore, Patrick W. Sink, Keith E. Stevens, Knox C. Theus, and Tony $\mathrm{D}$. Washington, for their valuable and tireless contributions to this project

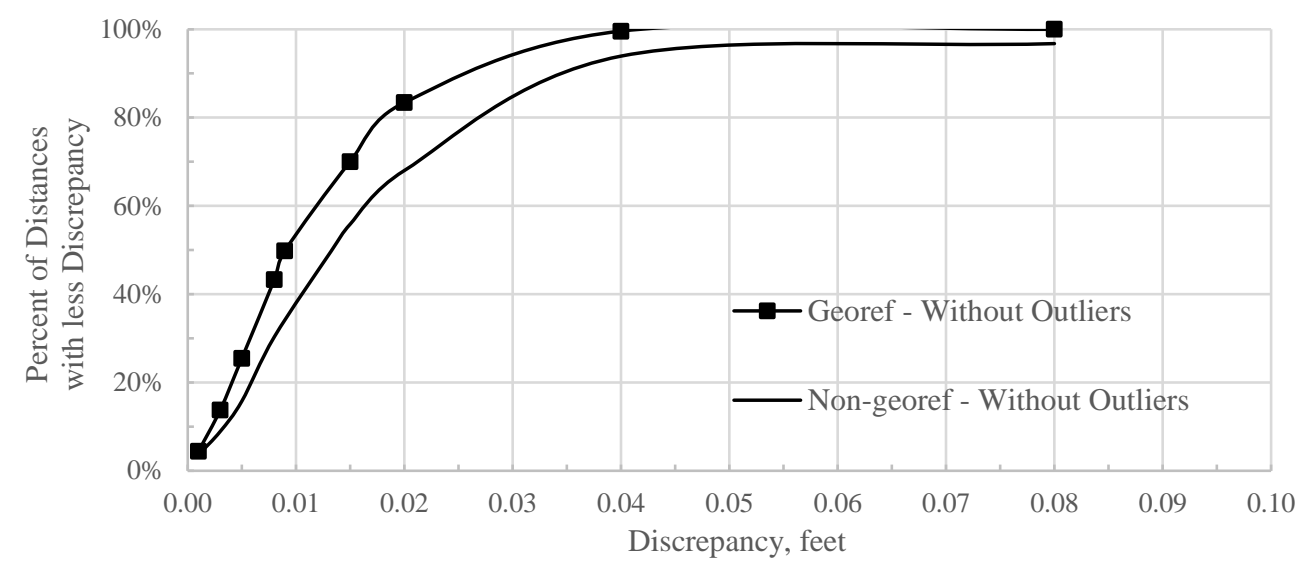

Figure 6. Discrepancies of georef and non-georef models (without outliers) vs field values (Cumulative Distribution Functions)

\section{References}

Holley, P.W., Perrine, T.H. and Gamble, T. (2011). Is Reflectorless EDM Technology Reliable for Building Construction Layout Tolerances? $47^{\text {th }}$ ASC Annual International Conference Proceedings.

Long, N.Q., Buczek, M.M., Hien, L.P. et al. (2018). Accuracy assessment of mine wall's surface models derived from terrestrial laser scanning. Int J Coal Sci Technol. Vol 5, Issue 3, p. 328-338.

Maldonado, G.O., Maghiar, M., Jackson, N.M., Garrett, D.M. and Givens, K.E. (2015). Comparison of Building Measurements Acquired via Laser-Based Scanner and Modern Total Station. 51 ${ }^{\text {st }}$ ASC Annual International Conference Proceedings.

Maldonado, G.O., Maghiar, M., Van Stan, J.T., Peart, M.D. and Mesta, D.C. (2018). Discrepancies in Measurements of a Complex City Intersection - Laser Scanner vs Accurate Robotic Total Station. $54^{\text {th }}$ ASC Annual International Conference Proceedings, p. 371-378.

Newsome, S.R. Jr (2016), Discrepancy Analysis Between Close-Range Photogrammetry and Terrestrial LiDAR" (2016). Digital Commons, Georgia Southern University, Electronic Theses and Dissertations. Document 1423.

Peart, M. D. (2019). Accuracy of 3D Point-Cloud and Photo-Based Models of City Street Intersections. Digital Commons, Georgia Southern University, Electronic Theses and Dissertations, Document 1943.

Slattery, K.T., Slattery, D.K., and Gordon, C. (2008). Evaluation of 3-D Laser Scanning for Highway Construction Applications. $44^{\text {th }}$ ASC Annual International Conference Proceedings. 